\title{
Cambio estructural en las economías de EI Salvador y Guatemala: un enfoque de la teoría de cointegración
}

\author{
Luis René Cáceres y Oscar A. Núnez Sandoval*
}

\section{Introducción}

Uno de los temas que más atención ha recibido en las ciencias económicas es el del cambio eslructural que ocurre en las economias en vías de desarrollo a medida que aumenla el ingreso percápita. Numerosos estudios econométricos han demostrado que el crecimiento económico da lugar a un cambio paulalino en la eslruclura de la producción. en términos de un aumento de la participación del sector manulaclurero en el produclo inlerno bruto lo cual ocurre simulláneamente a una disminución de la participación del sector agropecuario (Chenery, 1960). Olros esludios han planteado que la velocidad con que se da esle cambio estruclural depende del lamaño de los paises y de su orientación exportadora (Chenery y Taylor, 1968). Las razones expuestas para esta Iranslormación se fundamentan en el cambio en la estructura de la demanda, que revela una declinación del consumo de bienes alimenticios y un aumenlo en el de bienes industriales, de cuerdo con el patrón denominado la Ley de Engel.

No obstanle los avances en la literatura en este campo, surgen algu-

- Los autores son funcionarios, respectivamente, del Banco Interamericano de Desarrollo y del Banco Centroamericano de Integración Económica 
nas interrogantes sobre la validez de las conclusiones presentadas. Una se retiere a la posibilidad de que las series de tiempo utllizadas en los esludios lengan una varianza infinita, es declr, no sean estacionarias, en cuyo caso no es confiable el análisis de regresión (Granger y Newbold, 1974). Otra preocupación que surge está relacionada al hecho de que el cambio estruclural observado sea de una naluraleza lemporal o transitoria, volviendo posteriormente la economía a su relación de largo plazo o de equilibrio.

En este trabajo se analiza por medio de la melodología de cointegración la existencia de cambio estructural en las economias de $\mathrm{EI}$ Salvador y Guatemala, utilizando específicamente los casos de las relaciones entre la producción industrial y agropecuaria con el produclo interno bruto per cápila. Se analiza empíricamente si exisle una relación de equilibrio de largo plazo entre las producciones industrial y agrícola y el ingreso per cápita, utilizando datos anuales del periodo 1925-1984. La sección siguiente expone brevemente la técnica de cointegración, mientras la sección III presenta los resultados empiricos; el trabajo concluye con una serie de consideraciones finales.

\section{La teoría de colntegraclón y la existencla de camblo estructural en una economia}

La teoria de cointegración se puede asociar con la existència de una relación estable en la trayectoria a largo plazo de dos variables, en la cual sus series de liempo no divergen persistentemente entre si, y regresan a sus trayectorias de largo plazo después de ser sometidas a perturbaciones. Si esta asociación existe, se infiere que las variables son cointegradas.

En términos operativos el concepto de cointegración parte de la definición de dos clases de series de tiempo: una, las series cuya media, varianza y autocorrelación temporal son constantes, las cuales se denominan series con propiedades "estacionarias" llamándose series integradas de orden cero, ó $I(0)$. Olra, aquellas series que necesilan que se oblenga su primera diferencia para que adquieran la caracteristica de estacionalidad, y se les llama series integradas de orden uno, o sea l(1). En términos generales, si una serie necesita ser diferenciada d veces para volverse $1(0)$, se le denomina serie integrada de orden $d$. Las series I(0) muestran una tendencia a regresar a su valor promedio y flucluaan en lorno al mismo sin apartarse de él considerablemente. Su función de aulocorrelación disminuye rápidamente en la medida que aumentan los rezagos, dando poca ponderación a evenlos en el pasado distante y, de esa lorma, tienen una "memoria" finila. Por su parte, una serie I(1) osci- 
lará ampliamente y raramente retornará a valores previos'

En términos más formales, se puede dar la siguiente definición de la propiedad de cointegración: considérese a $X, Y$ un par de series de orden 1, entonces. si existe una constante $A$, lal que $Z=X-A Y$ sea de orden cero, se infiere que $X$ y $Y$ son cointegradas y $A$ se denomina el parámetro de cointegración. La expresión $X=A Y$ se puede interpretar como una relación de largo plazo o de "equilibrio" $y$, de esa manera, la variable $Z$, que refleja el grado en que las variables $X$ y $Y$ se apartan de tal relación, se le conoce como el "error de equilibrio".

Una extensión de la leoria es que si $X$ y $Y$ son $l(1)$ y son coinlegradas, enlonces existe un mecanismo de "correlación de errores" definido de la siguiente manera:

$$
\begin{aligned}
& \Delta X=-\sigma_{1} Z_{-1}+\sum_{i=1}^{n} \alpha_{1} \Delta X_{-1}+\sum_{i=1}^{n} \beta_{i} \Delta Y_{-1}+\epsilon_{1} \\
& \Delta Y=-\sigma_{2} Z_{-1}+\sum_{i=1}^{n} \delta_{i} \Delta X_{-1}+\sum_{i=1}^{n} \theta_{i} \Delta Y_{-1}+\epsilon_{2}
\end{aligned}
$$

En donde $Z=X-A Y, y$ los errores $\varepsilon_{1}$ y $\varepsilon_{2}$ tienen la caracteristica de "ruido blanco".

Granger (1986) y Engle y Granger (1987) han demostrado que si dos variables son cointegradas entonces debe comportarse de acuerdo al modelo de corrección de errores y debe existir una relación de causalidad, en el sentido de Granger, en por to menos una dirección, dado que una variable sirve para pronoslicar el valor de la otra.

La melodologia de coinlegración se ha aplicado para detectar la exislencia de relaciones de largo plazo entre variables macroeconómicas; en este sentido, cabe destacar los estudios efectuados para comprobar la validez de la teoría de la paridad del poder de compra mediante la invesligación de la existencia de coinlegración entre los precios domésticos y externos, ajustando eslos úllimos por el lipo de cambio; el análisis de la relación entre el ingreso y consumo de energía per capita; la relación entre la lasa de interés nacional y la tasa de inflación; y las relaciones entre dinero e ingreso; impuestos y gastos público; déficit fiscales, lasa de interés y tasas de cambio.

1. Las referencias clásicas de la teoría de coinlegración son Granger (1983), y Engle y Granger (1987). Una completa exposición en castellano se encuentra en Chica Avella (1990). 


\section{Prueba de colntegración para determinar el camblo estructural}

En este trabajo la aplicación de la metodologia de cointegración al estudio del cambio estructural radica en delectar si las series de liempo de la producción industrial y agrícola son coinlegradas con la serie del producto interno brulo per cápita. Si se encuentran evidencias de cointegración se puede inferir que exisle una asociación estable de largo plazo, enlre ambas variables, aunque se presenten periodos en que las variables se separan o acercan, rompiendo temporalmente la asociación de largo plazo; el punto de importancia es que estos "disturbios" no son permanentes y las variables regresan a su trayectoria estable de largo plazo.

A continuación se investiga la hipótesis de cointegración entre la producción industrial y agropecuaria con el ingreso per cápita. En adición, se explora la existencia de cointegración entre los términos de intercambio y el ingreso per cápita. Se han usado dalos anuales para El Salvador y Gualemala, por un periodo relativamente largo de 1925 a 1984 reportados por Bulmer-Thomas (1990).

Siguiendo a Engle y Granger (1987) el análisis se efectúa en Ires elapas:

Paso 1:Es necesario conocer si las variables producción industrial y agropecuaria, términos de intercambio e ingreso per cápila de El salvador y Gualemala son estacionarias. Para este propósito se recurre a la prueba desarrollada por Dickey y Fuller (1979) que en términos generales, para el caso de una variable $X$, requiere la estimación de la ecuación:

$$
\Delta X_{i}=\sigma X_{i-1}+\sum_{i=1}^{p} \theta_{i} \Delta X_{t-i}+e t
$$

En donde el tamaño del rezago $p$ se selecciona para asegurar que et es "ruido blanco."2

La hipótesis nula, $H_{0}: X_{t}$ es $I(1)$ se rechaza en lavor de $H_{1}: X_{1}$ es $I(0)$, si se encuentra que $\sigma$ es negativo y estadisticamente significativo. La prueba se realiza calculando un "estadístico l" igual a la razón del valor del coelicienle $ø$ a su error estándar. Este estadíslico es comparado con los

2. Para seleccionar $p$ se usa el criterio del estadístico $Q$, de Box-Pierce, el cual se calcula con las autocorrelaciones de los residuos y presenta una distribución Chi-cuadrado. 
valores críticos computados por Dickey y Fuller (1979), ya que no tiene una distribución 1 en visla de que la varianza de $X$ es inlinita si la hipótesis nula es aceplada.

Los resultados de la eslimación de la ecuación (2) para los logaritmos de las variables correspondientes a EI Salvador, demuestran que las cuatro variables no son eslacionarias, tal como se presenta en el Cuadro 1, en donde se puede observar que en todas las estimaciones el "estadistico I" de $\varnothing$ es inferior al valor crílico de 2.89 a un nivel de 5 por ciento. De esa manera, se infiere que las variables Yes, Yies, Yaes y Ties son I(1), cumpliendo asi una condición necesaria para la existencia de cointegración.

En el cuadro 2 se presentan los resullados correspondientes a Gua-

Cadro 1

Resultado para el caso de el Salvador*

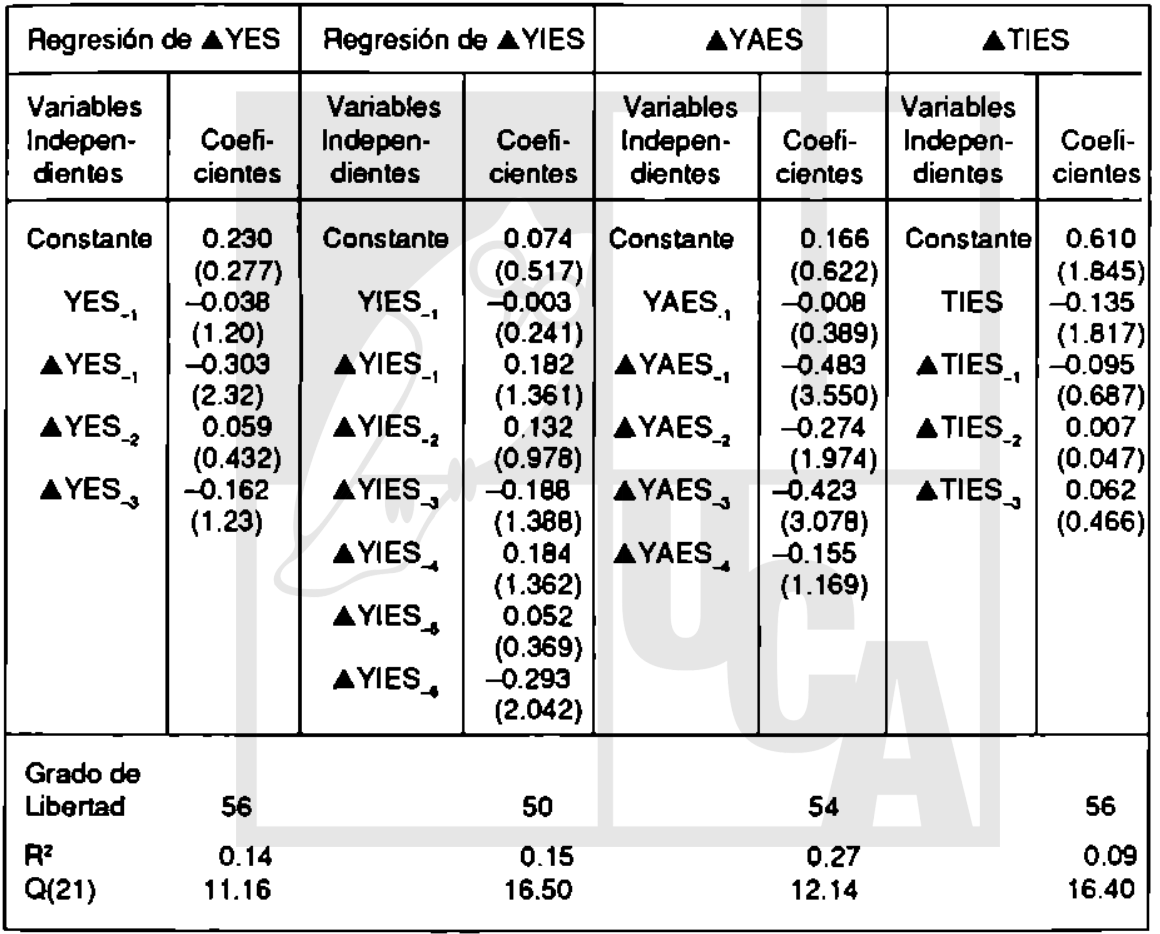

- Las variables se expresen en logaritmos y son las siguientes:

YES: Producto Interno Bruto Percapita a precios de 1970

YIES: Valor Agregado en Manulactura a precios de 1970

YAES: Valor Agregado en Agricultura a precios de 1970

TIES: Términos Netos de Intercambio, $1970=100$ 


\section{Cadro 2}

Resultado para el caso Guatemala

\begin{tabular}{|c|c|c|c|c|c|c|c|}
\hline \multicolumn{2}{|c|}{ Regresión de $\Delta$ YG } & \multicolumn{2}{|c|}{ Regresión de $\Delta Y I G$} & \multicolumn{2}{|c|}{$\triangle Y A G$} & \multicolumn{2}{|c|}{$\Delta$ TIG } \\
\hline $\begin{array}{l}\text { Variables } \\
\text { Indepen- } \\
\text { dientes }\end{array}$ & $\begin{array}{l}\text { Coefi- } \\
\text { cientes }\end{array}$ & $\begin{array}{l}\text { Variables } \\
\text { Indepen- } \\
\text { dientes }\end{array}$ & $\begin{array}{l}\text { Coeli- } \\
\text { cientes }\end{array}$ & $\begin{array}{l}\text { Variables } \\
\text { Indepen- } \\
\text { dientes }\end{array}$ & $\begin{array}{l}\text { Coefi- } \\
\text { cientes }\end{array}$ & $\begin{array}{l}\text { Variables } \\
\text { Indepen- } \\
\text { dientes }\end{array}$ & $\begin{array}{c}\text { Coeli- } \\
\text { cientes }\end{array}$ \\
\hline Constante & 0.278 & Constanle & 0.074 & Constante & 0.220 & Constante & 0.533 \\
\hline YG & $\begin{array}{c}(1.177) \\
-0.046\end{array}$ & YIG & $\begin{array}{c}(0.468) \\
0.011\end{array}$ & YAG & $\begin{array}{c}(0.819) \\
-0.015\end{array}$ & TIG & $\begin{array}{c}(1.738) \\
-0.121\end{array}$ \\
\hline-1 & (1.129) & - & (0.793) & ו- & (0.692) & 10 & $(1.772)$ \\
\hline$\Delta Y G_{-1}$ & $\begin{array}{c}0.249 \\
(1.964)\end{array}$ & $\Delta Y I G_{-1}$ & $\begin{array}{c}0.034 \\
(0.279)\end{array}$ & $\triangle Y A G_{-1}$ & $\begin{array}{c}0.096 \\
(0.737)\end{array}$ & $\Delta T I G_{-1}$ & $\begin{array}{c}0.079 \\
(0.650)\end{array}$ \\
\hline & $\begin{array}{c}-0.026 \\
(0.202)\end{array}$ & $\Delta Y I_{-2}$ & $\begin{array}{c}-0.133 \\
(1.131)\end{array}$ & $\triangle Y A G_{-2}$ & $\begin{array}{c}0.0815 \\
(0.618)\end{array}$ & $\Delta T^{T} G_{-2}$ & $\begin{array}{c}0.196 \\
(1.635)\end{array}$ \\
\hline$\Delta Y G_{\rightarrow}$ & $\begin{array}{c}-0.233 \\
(1.822)\end{array}$ & $\Delta Y I G_{\vec{\nu}}$ & $\begin{array}{c}-0.242 \\
(2.008)\end{array}$ & $\triangle Y A^{-3}$ & $\begin{array}{l}-0.158 \\
(1202)\end{array}$ & $\Delta T I G_{-3}$ & $\begin{array}{c}0.174 \\
(1.425)\end{array}$ \\
\hline & & & & & & $\Delta T_{1} G_{-A}$ & $\begin{array}{c}-0.976 \\
(0.828)\end{array}$ \\
\hline $\begin{array}{l}\text { Grado de } \\
\text { Libertad }\end{array}$ & 56 & & 56 & & 56 & & 54 \\
\hline $\begin{array}{l}\mathrm{R}^{2} \\
\mathrm{Q}(21)\end{array}$ & $\begin{array}{r}0.16 \\
11.90\end{array}$ & & $\begin{array}{r}0.09 \\
13.49\end{array}$ & & $\begin{array}{r}0.05 \\
27.50\end{array}$ & & $\begin{array}{r}0.13 \\
12.27\end{array}$ \\
\hline
\end{tabular}

- Las variables se expresan en logaritmos y son las siguienles:

YG: Producto Interno Bruto Percápita a Precios de 1970

YIG: Valor Agregado en Manulactura a precios de 1970

YAG: Valor Agregado en Agricultura a precios de 1970

TIG: Términos Netos de Intercambio, $1970=100$

temala, pudiéndose nolar que ninguna variable es eslacionaria.

Los resullados de los cuadros anteriores permiten aceptar la hipótesis nula, $H 0$ : $X_{Y}$ es I(1), para todas las series de los dos países. De esta manera, se cumple una condición necesaria para continuar con el proceso de la prueba de cointegración.

Paso 2: Usando mínimos cuadrados ordinarios se estima la ecuación de cointegración siugiente:

$$
X_{1}=A+B Y_{1}+\mu_{1}
$$

El resultado de la eslimación de la ecuación (3) para El Salvador se reporta a continuación: 
Cuadro 3

Ecuación de cointegración para el caso de El Salvador

\begin{tabular}{|c|c|c|c|c|c|}
\hline & & & & $\overline{\mathrm{A}^{2}}$ & D.W. \\
\hline YIES $=$ & $\begin{array}{l}=-3.55 \\
(6.48)\end{array}$ & $\begin{array}{r}2.66 \\
(27.15)\end{array}$ & YES & 0.92 & 0.68 \\
\hline YAES = & $=\begin{array}{r}3.40 \\
(10.08)\end{array}$ & $\begin{array}{cc}1.60 \\
(2.65)\end{array}$ & YES & 0.92 & 0.40 \\
\hline TIES $=$ & $=\begin{array}{c}0.018 \\
(0.033)\end{array}$ & $\begin{array}{r}0.797 \\
(8.12)\end{array}$ & YES & 0.50 & 0.64 \\
\hline
\end{tabular}

Para el caso de Guatemala los resultados son los siguientes:

\section{Cuadro 4}

Ecuaclón de cointegraclón para el caso de Guatemala

\begin{tabular}{|c|c|c|c|c|}
\hline & & & $\mathrm{R}^{2}$ & D.W. \\
\hline YIG & $=\begin{array}{c}-4.43 \\
(-4.54)\end{array}$ & $+\begin{array}{r}2.80 \\
(16.65)\end{array} \quad Y G$ & 0.81 & 0.30 \\
\hline YAG & $\begin{array}{l}=-1.17 \\
(2.59)\end{array}$ & $+\underset{(31.03)}{2.41}$ YG & 0.94 & 0.48 \\
\hline TIG & $\begin{array}{r}=\quad 4.28 \\
(4.67)\end{array}$ & $+\begin{array}{c}0.028 \\
(0.18)\end{array} \quad Y G$ & -0.02 & 0.22 \\
\hline
\end{tabular}

Paso 3 Se comprueba que el error $\mu_{1}$ de la ecuación (3) sea eslacionario. La exislencia de cointegración es aceptada cuando el residuo $\mu$ liene la propiedad $\mathrm{I}(0)$, es decir que es estacionario. Para este fin se recurre a las siguientes pruebas:

Prueba 1: El estadístico Durbin-Walson de la Regresión de Cointegración (DWRC).

De acuerdo a Sargan y Bhargava (1983), si el esladistico DurbinWalson de la regresión de cointegración es signiticalivamente mayor que cero, se puede rechazar la hipólesis de que no existe cointegración. La significancia estadística del estadístico Durbin-Watson oblenido al estimar la ecuación (3) se establece utilizando los valores crítico reporlados en Engle y Granger (1987), para una muestra de 100 observacio- 
nes; si el estadistico DW es mayor que los valores crílicos se acepta la existencia de cointegración.

Los valores crilicos reportados en Engle y Granger (1987) para el estadistico Durbin-Watson son de $0.511,0.386$ y 0.322 a los niveles de $1 \%, 5 \%$ y $10 \%$ respectivamente. De esa manera, para el caso de El Salvador se puede inferir que se acepta la prueba de coinlegración a un nivel de $1 \%$ en los casos de producción industrial y lérminos de intercambio con el producto interno bruto, mientras que a un nivel del $5 \%$ se acepla la cointegración entre producción agropecuaria e ingreso per cápita.

En el caso de Guatemala se puede notar en el Cuadro 4 que la existencia de cointegración sólo se puede aceptar, y a un nivel de $5 \%$, en el caso de la producción agropecuaria con el ingreso per cápita.

Prueba 2: El estadístico de Dickey-Fuller (DF)

Utilizando la serie de los residuos de la ecuación de regresión (3) se estima una ecuación propuesla por Dickey y Fuller (1979):

$$
\Delta \mu_{t}=\delta \mu_{t-1}+v_{1}
$$

La significancia esladística del parámetro $\delta$ se eslablece usando su "estadístico 1", comparándolo con los valores crílicos de Engle y Granger (1987). Si el "estadistico 1" de $\delta$ es mayor que los valores críticos se acepla la hipótesis de existencia de cointegración.

Los resullados para El Salvador y Gualemala se muestran en los Cuadros 5 y 6 , respectivamente. Los valores crílicos para la significancia de $\delta$ son de $4.07,3.37$ y 3.03 a niveles respectivos de $1 \%, 5 \%$ y $10 \%$. Por lo tanto, se puede interir que para el caso de la producción industrial e ingreso per cápita de El Salvador existe cointegración (a un nivel de significancia esladistica del $10 \%$ ), mientras que a un nivel del $5 \%$ se acepta la hipólesis de coinlegración para el caso de los términos de inlercambio y el ingreso. Además, el estadístico $Q$ de todas las regresiones es menor que el valor crítico de la distribución chi-cuadrado, al $95 \%$ de nivel de probabilidad, indicando que no exisle autocorrelación de residuos. 


\section{Cuadro 5}

Regreslones de la prueba de colntegración

para el caso de El Salvador

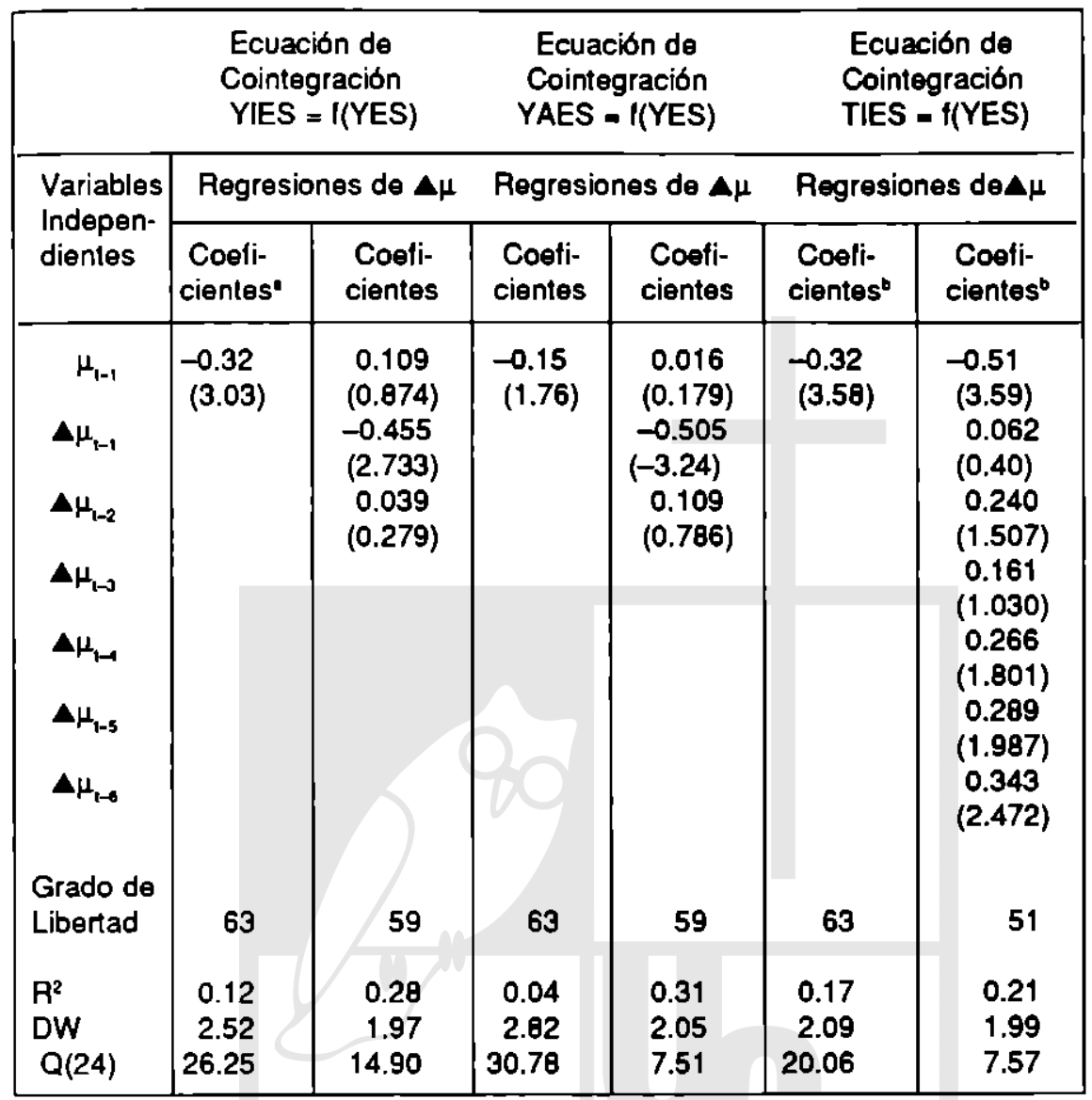

a. Se acepla la existencia de cointegración a un nivel de 10 por ciento.

b. Se acepla la existencia de cointegración a un nivel de $\mathbf{5}$ por ciento.

Como se puede notar en el Cuadro 6, en el caso de Guatemala no se encuentra coinlegración entre ninguno de los pares de variables.

Prueba 3: el estadístico de Dickey-Fuller aumentado (DFA)

De nuevo, con los residuos de la regresión (3) se estima una ecuación que incorpora valores rezagados de $\Delta \mu$ a fin de asegurar que el error $\mu_{1}$ es ruido blanco y tiene la forma siguiente: 
Cuadro 6

Regresiones de la prueba de cointegración para el caso de Guatemala

\begin{tabular}{|c|c|c|c|c|c|c|}
\hline & \multicolumn{2}{|c|}{$\begin{array}{l}\text { Ecuación de } \\
\text { Cointegración } \\
Y I G=f(Y G)\end{array}$} & \multicolumn{2}{|c|}{$\begin{array}{l}\text { Ecuación de } \\
\text { Cointegración } \\
Y A G=f(Y G)\end{array}$} & \multicolumn{2}{|c|}{$\begin{array}{l}\text { Ecuación de } \\
\text { Cointegración } \\
\text { TIG }=1(Y G)\end{array}$} \\
\hline \multirow{2}{*}{$\begin{array}{l}\text { Variables } \\
\text { Indepen- } \\
\text { dienles }\end{array}$} & \multicolumn{2}{|c|}{ Regresiones de $\Delta_{\mu}$} & \multicolumn{2}{|c|}{ Regresiones de $\Delta \mu$} & \multicolumn{2}{|c|}{ Regresiones de $\Delta \mu$} \\
\hline & $\begin{array}{l}\text { Coefi- } \\
\text { cientes }\end{array}$ & $\begin{array}{l}\text { Coefi- } \\
\text { cientes" }\end{array}$ & $\begin{array}{l}\text { Coofi- } \\
\text { cientes }\end{array}$ & $\begin{array}{l}\text { Coefi- } \\
\text { cientes }\end{array}$ & $\begin{array}{l}\text { Coefi- } \\
\text { cientes }\end{array}$ & $\begin{array}{c}\text { Coefi- } \\
\text { cientes }\end{array}$ \\
\hline $\begin{array}{c}\mu_{1-1} \\
\Delta \mu_{1-1} \\
\Delta \mu_{1-2}\end{array}$ & $\begin{array}{c}-0.14 \\
(2.02)\end{array}$ & $\begin{array}{c}0.24 \\
(3.17) \\
0.33 \\
(2.67) \\
0.18 \\
(1.36)\end{array}$ & $\begin{array}{c}-0.24 \\
(2.79)\end{array}$ & $\begin{array}{c}-0.35 \\
(3.44) \\
0.37 \\
(3.05) \\
0.05 \\
(0.41)\end{array}$ & $\begin{array}{c}-0.09 \\
(1.63)\end{array}$ & $\begin{array}{c}-0.171 \\
(2.75) \\
0.17 \\
(1.41) \\
0.16 \\
(1.29)\end{array}$ \\
\hline $\begin{array}{l}\text { Grado de } \\
\text { Libertad }\end{array}$ & 63 & 59 & 63 & 59 & 63 & 59 \\
\hline $\begin{array}{l}\mathrm{R}^{2} \\
\mathrm{DW} \\
\mathrm{Q}(24)\end{array}$ & $\begin{array}{r}0.06 \\
1.42 \\
22.80\end{array}$ & $\begin{array}{r}0.178 \\
1.97 \\
10.74\end{array}$ & $\begin{array}{r}10.6 \\
1.44 \\
34.50\end{array}$ & $\begin{array}{l}0.19 \\
1.77 \\
23.3\end{array}$ & $\begin{array}{r}0.04 \\
1.54 \\
12.10\end{array}$ & $\begin{array}{r}0.10 \\
1.98 \\
15.80\end{array}$ \\
\hline
\end{tabular}

a. Se acepta la existencia de coinlegración a un nivel de 10 por ciento.

b. Se acepla la existencia de cointegración a un nivel de 5 por ciento.

$$
\mu_{1}=\Phi \mu_{1-1}+\sum \underset{i=1}{\tau_{1}} \nabla_{\mu_{i-1}}+w_{1}
$$

Es del caso sefialar que Engle y Granger (1987) recomendaron el uso del esladístico DFA como el más apropiado en la prueba de cointegración. En el Cuadro 5 se observa que para El Salvador sólo se encuentra la exislencia de cointegración entre las series lérminos de intercambio e ingreso per cápita. En el cuadro 6 se puede nolar que en Guatemala la cointegración es aceptada a un nivel de $10 \%$ para el caso de las series producción industrial e ingreso y a un nivel de $5 \%$ para la producción agricola e ingreso. 


\section{Modelo de correcclón de errores}

En la sección anterior se encontró evidencia de que la producción agricola, industrial y los términos de intercambio de El Salvador son cointegrados con el ingreso percápita. Asimismo, en el caso de Guatemala se encontró que la producción industrial y agricola son cointegrados con el ingreso por habitante. Lo anterior indica que debe existir un módulo de corrección de errores para estas variables.

Modelos de corrección de errores fueron estimados para las variables dependienles $\triangle$ YES, $\triangle$ YIES, $\triangle$ YAES Y $\triangle$ TIES. Se debe notar que la ecuación para $\triangle$ YIES usa el término de error de la ecuación de cointegración de YIES explicada por YES, y viceversa, y que este término de error con un rezago de un ano representa la respuesla de la variable dependiente a las desviaciones del equilibrio. Si el término de error no es significativo se deduce que la variable dependiente no se ajusta para corregir las desviaciones del equilibrio.

Los resultados correspondientes a los casos de YIES y YES se presentan en los cuadros 7 y 8 . Se puede apreciar que el término de error rezagado es significativo en la ecuación que explica $\triangle Y E S$ y no es significativo cuando $\triangle Y E S$ es la variable dependiente. Esto indica que el ingreso percápila causa la producción induslrial. Otros resultados (otros resullados que no se muestran) indican que existe una relación de causalidad en ambas direcciones entre YIES y YAES, asi como consolidad en una dirección de YES a los términos de intercambio.

\section{Consideraciones finales}

Los resullados anleriores han demostrado, en primer lugar, que todas las series analizadas son integradas de orden uno, 10 cual indica que se debe tener mucho cuidado en interprelar los resultados de análisis de regresión usando estas series, ya que los resultados pueden ser espurios.

El análisis de cointegración indica que en El Salvador usando las tres pruebas realizadas se puede inferir que los términos de intercambio y el ingreso per cápita son cointegrados, es decir, mantienen una relación estable de largo plazo o sea que El Salvador es tan vulnerable a las visicitudes de los términos de intercambio en los anos ochenta como lo fue en los años veinte. Además, usando las pruebas basadas en el estadístico Durbin-Walson y el estadístico de Dick-Fuller, se encuentra evidencia de cointegración enlre la producción induslrial y el ingreso per cápila. Eslo indica que no ha ocurrido un "cambio estructural" sino que la relación entre eslas dos variables se han mantenido eslable a to largo 


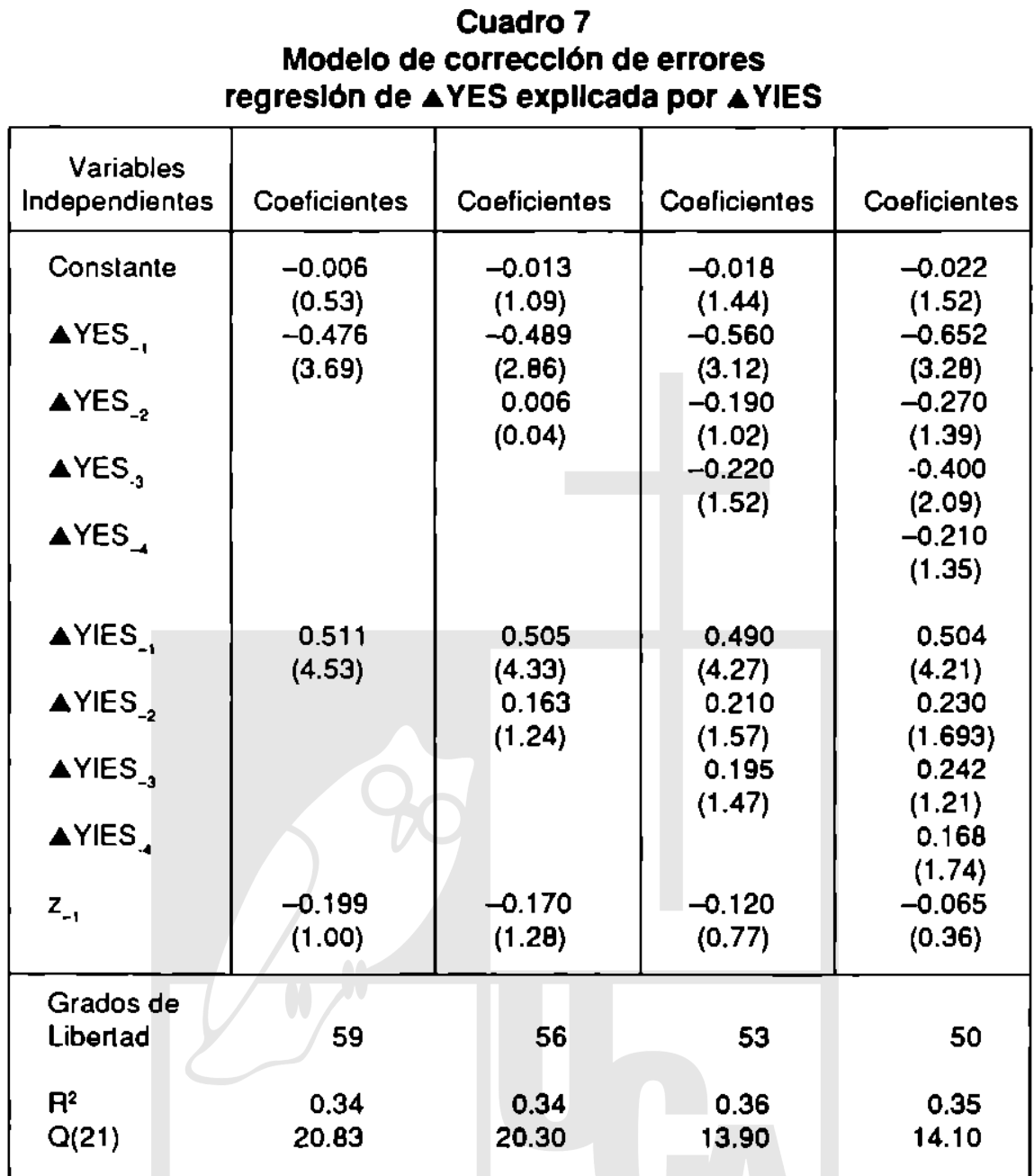

de las 5 décadas analizadas. Además, en base a la prueba del estadístico DW también se nota la existencia de cointegración entre la producción agrícola y el ingreso. Debe senalarse que los estudios de cambio estructural en la literatura han sido de tipo de corte transversal, sin analizar paises individuales a lo largo del liempo.

En el caso de Guatemala se encontró la exislencia de cointegración entre la producción industrial y el ingreso per cápila de acuerdo a los esladísticos DW y el de Dick-Fuller aumentado. En base a esla última prueba lambién se enconiró que la producción agricola y el ingreso son 


\section{Cuadro 8 \\ Modelo de corrección de errores \\ regresión de $\triangle$ YIES explicada por $\triangle$ YES}

\begin{tabular}{|c|c|c|c|c|}
\hline $\begin{array}{c}\text { Variables } \\
\text { Independienles }\end{array}$ & Coeficienies & Coeficientes & Coeficientes & Coeficientes \\
\hline Constante & $\begin{array}{r}0.035 \\
(2.86)\end{array}$ & $\begin{array}{l}0.033 \\
(2.44)\end{array}$ & $\begin{array}{r}0.039 \\
(2.78)\end{array}$ & $\begin{array}{l}0.032 \\
(1.98)\end{array}$ \\
\hline$\Delta Y I E S_{-1}$ & $\begin{array}{r}0.177 \\
(1.34)\end{array}$ & $\begin{array}{r}0.224 \\
(1.63)\end{array}$ & $\begin{array}{r}0.231 \\
(1.78)\end{array}$ & $\begin{array}{l}0.232 \\
(1.73)\end{array}$ \\
\hline$\Delta Y I E S_{-2}$ & & $\begin{array}{c}0.109 \\
(0.705)\end{array}$ & $\begin{array}{r}-0.197 \\
(1.31)\end{array}$ & $\begin{array}{l}0.213 \\
(1.39)\end{array}$ \\
\hline$\triangle Y^{\prime E S}{ }_{3}$ & & & $\begin{array}{c}-0.042 \\
(0.28)\end{array}$ & $\begin{array}{l}0.005 \\
(0.02)\end{array}$ \\
\hline$\triangle Y I E S_{-}$ & & & & $\begin{array}{r}0.174 \\
(1.13)\end{array}$ \\
\hline$\triangle Y E S_{-1}$ & $\begin{array}{l}-0.162 \\
(1.066)\end{array}$ & $\begin{array}{c}-0.366 \\
(1.77)\end{array}$ & $\begin{array}{c}-0.547 \\
(2.65)\end{array}$ & $\begin{array}{c}-0.695 \\
(2.51)\end{array}$ \\
\hline$\triangle Y E S_{-2}$ & & $\begin{array}{l}-0.259 \\
(1.49)\end{array}$ & $\begin{array}{l}-0.539 \\
(2.51)\end{array}$ & $\begin{array}{l}-0.607 \\
(2.55)\end{array}$ \\
\hline$\triangle Y E S_{-3}$ & & & $\begin{array}{l}-0.409 \\
(2.44)\end{array}$ & $\begin{array}{l}-0.540 \\
(2.35)\end{array}$ \\
\hline$\triangle Y^{\prime} S_{4}$ & & & & $\begin{array}{r}-0.126 \\
(0.69)\end{array}$ \\
\hline$z_{-1}$ & $\begin{array}{r}-0.094 \\
(1.82)\end{array}$ & $\begin{array}{c}-0.033 \\
(2.20)\end{array}$ & $\begin{array}{r}-0.219 \\
(3.36)\end{array}$ & $\begin{array}{c}-0.220 \\
(2.89)\end{array}$ \\
\hline $\begin{array}{l}\text { Grados de } \\
\text { Libertad }\end{array}$ & 59 & 56 & 53 & 50 \\
\hline $\begin{array}{l}\mathrm{R}^{2} \\
\mathrm{Q}(21)\end{array}$ & $\begin{array}{r}0.03 \\
25.35\end{array}$ & $\begin{array}{r}0.04 \\
28.80\end{array}$ & $\begin{array}{r}0.13 \\
23.90\end{array}$ & $\begin{array}{r}0.12 \\
18.20\end{array}$ \\
\hline
\end{tabular}

cointegrados. Estos resullados también dan motivos para dudar de la existencia de un "cambio estructural" en la economía guatemalteca.

Se debe seffalar que el aumento de la participación de la industria dentro del PIB se ha explicado en un 50\% como consecuencia de la ley de Engel y el otro $50 \%$ es causado por la sustitución de imporlaciones y el crecimiento de las exporlaciones de manufacturas (Chenery y Syrquin, 1975). Pareciera entonces que estos dos últimos fenómenos han eslado ausentes en las economias de El Salvador y Guatemala, a la vez que el efecto de la ley de Engel, o sea el incremenlo en la 
demanda de manulacluras a medida que aumenta el ingreso, pudo haber sido atendido mediante importaciones. Surge, además, la conclusión que en estos paises lodavia no ha ocurrido la transición hacia el "crecimiento económico moderno" definido en Chenery y Syrquin (1975, pág. 135), como: "un conjunto de cambios estructurales que han acompanado casi siempre al crecimiento del ingreso per cápila en décadas recientes. La mayoría de estos rasgos ya han sido identificados en la historia de los palses industrializados, que Kuznets considera como crecimienlo económico moderno".

Los resultados anteriores podrian guardan relación con los de CheneRy y Taylor, quienes encontraron que en las economias pequenas (en lérminos de población) y orientadas a la exportación de bienes primarios, el cambio eslructural viene relativamente tarde en el proceso de desarrollo. Esto lo ha expresado Yotopoulos y Nugent (1976) en los términos siguientes:

"Al enfatizar las exportaciones primarias los paises pequenos orientados a la producción de bienes primarios no sólo tienden a retardar o mitigar el patrón normal de cambio sectorial, sino a revertirlo por cierto período de tiempo... Los países pequenos abiertos al comercio intemacional y con dotaciones de factores que les dan ventajas comparativas en el sector primario no son propensos a experimentar el tipo de cambios estruclurales asociados normalmenle con el desarrollo."

Lo anterior pone de relieve la imporlancia que el programa de integración económica liene para los países centroamericanos, como un medio de superar la limilación que impone su reducido tamaño. Los resultados obtenidos en este Irabajo muestran la necesidad de profundizar en el análisis de largo plazo de las economias en vías de desarrollo para conocer la invariabilidad de su estructura de producción y de su vulnerabilidad a la economia internacional.

\section{REFERENCIAS BIBLIOGRAFICAS}

Bulmer-Thomas, Victor The Political Economy of Central America since 1920, Cambridge University Press, 1987.

Chenery, H.B., "Patlerns of Industrial Growth", American Econiomic Review, Vol. 50, septiembre 1960, pp. 624-654.

Chenery, H.B. y L.J. Taylor, Development Palterns Among Countries and Over Time". The Review of Economic and Statistics, Vol. 50, noviembre ç1968, pp. 391-416.

Chenery, H.B. y Moises Syrquin, Patterns of Development 1950-1970. New York, Oxlord Universily Press, 1975.

Chica Avella, Ricardo, "La Metodología de la cointegración: Presentación y Algunas Aplicaciones", Desarrollo y sociedad, No. 25, marzo 1990, pp. 57-85. 
Dickey, D.A., y W.A. Fuller, Distribution of the Estimators for AutorregressiveTime Series with a Unit Rool". Journal of the Amercan Statistical Association 74 (1979): 427-31.

Engle, R.F. y C.W.J. Granger. "Co-inlegración and Error Correction: Representation, Estimation, and Tesling". Econométrica 55, 1987 pp. 251. 76.

Granger C.W.J., "Co-integrated Variables and Error-Correcling Models". Discussion Paper, 83-13, UCSD, 1983.

Granger, C.W.J., "Development in the Study of Cointegrated Economic Variables:" Oxford bulletin of Economics and Statistics, Vol. 48, No. 3, 1986, pp. 213-227.

Granger, C.W.J., y P. Newbold, "Spurious regressions in econometries", Journal of econometrics, Vol. 2, 1974, pp. 111-120.

Sargan, J.D., y A. Bhargava, "Testing Residuals from Least Squares regresion for Being generated by the Gaussian. Random Walk", Econométrica, 51, 1983, pp. 153-174.

Yolopoulos Pau a. y Jellrey b. Nugent, Economics of development, New York, Harper and row, 1976. 